\title{
Deux Nématodes parasites d'Amphibiens du Togo
}

\author{
par Marie-Claude DURETTE-DESSET * et Guéorgui BATCHAROV ** \\ * Laboratoire de Zoologie (Vers) associé au C.N.R.S. ( $\mathrm{P}^{r}$ A.-G. Chabaud) \\ Muséum national d'Histoire naturelle, 43, rue Cuvier, F 75231 Paris Cedex 05 \\ ** Chaire d'Ecologie, Université P. Hilendarski \\ Plovdiv, ul. Vassil Markov 7, Bulgarie
}

\section{Résumé.}

1) Description de la femelle de Gendria leberrei, Bain et Philippon, 1969, et compléments morphologiques à l'étude du mâle.

2) Description de Camallanus dimitrovi n. sp., parasite de divers: Amphibiens du Togo, caractérisé par son nombre élevé de baguettes chitinoïdes.

Les 13 espèces connues de Camallanus d'Amphibiens sont caractérisées par 4 éléments que l'on rencontre plus fréquemment que chez les Camallanus de Poissons (groupement des 3 premières paires de papilles postcloacales, forme du spicule droit, position preéquatoriale de la vulve, viviparité). Bien qu'il nous semble prématuré de créer un nouveau sousgenre pour les Camalianus d'Amphibiens, nous constatons qu'ils forment un groupe à part, plus évolué que celui des Poissons.

\section{Summary.}

Two Nematodes parasites of Amphibian from Togo.

1) Description of the female of Gendria leberrei Bain and Philippon, 1969, and complements to the morphological study of the male.

2) Description of Camallanus dimitrovi n.sp., a parasite of several Amphibians from Togo, characterized by large number of chitinous rods.

The 13 known species of Camallanus of Amphibians have four main morphological characteristics which are present more frequently than in Camallanus of fishes (position of the three first postcloacal papillae which are close to each other, shape of the right spicule, preequatorial position of the vulva, viviparity). More information is needed before a new sub-genus is created for the Camallanus of Amphibians but they already appear to form a separate group, more evolved than the Camallanus of fishes. 

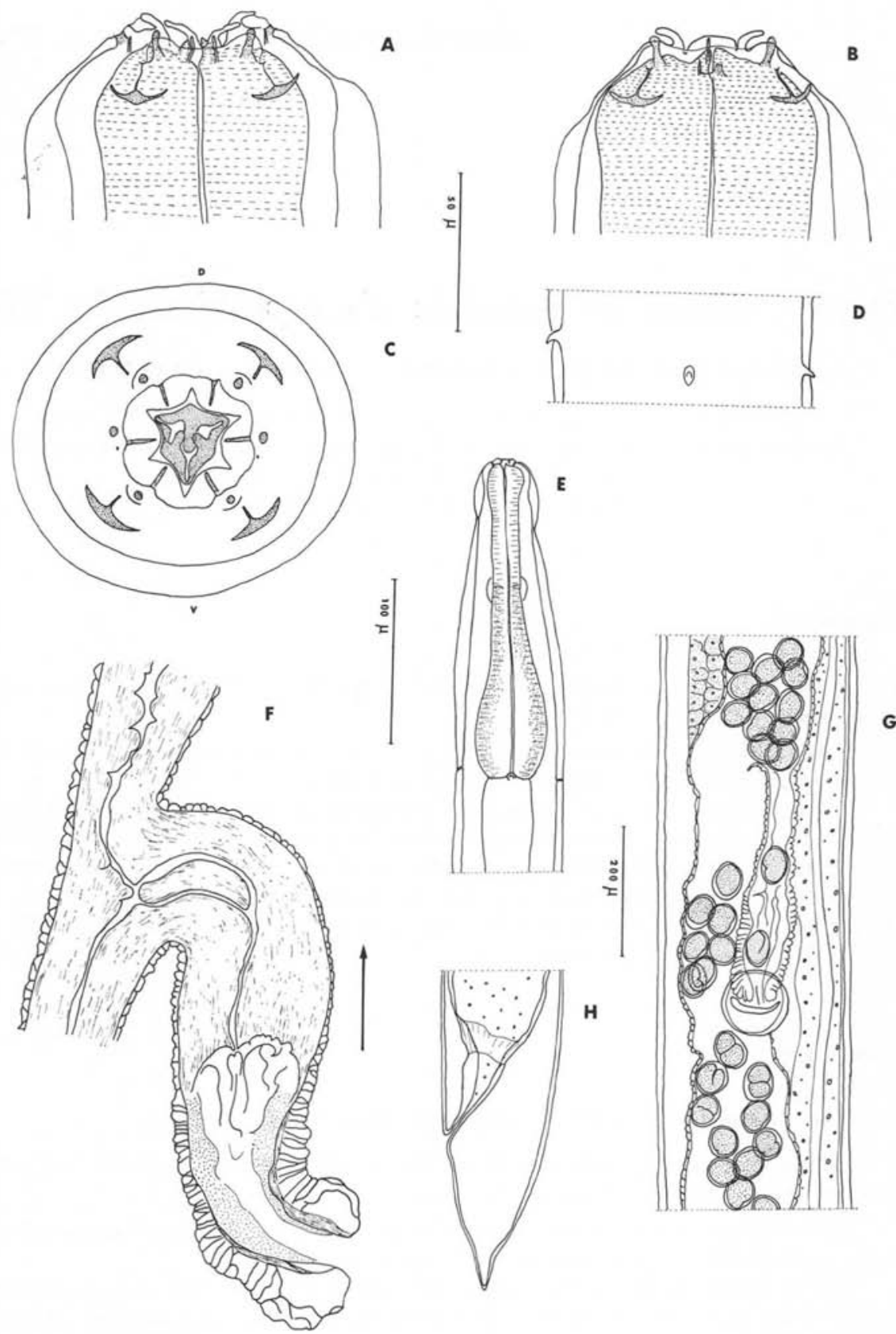

Fig. 1. - Gendria leberrei. Bain et Philippon, 1969, ơ A, B, C, tête, successivement en vues ventrale, latérale gauche et apicale. $\mathrm{D}$, détail du pore excréteur et des deirides, vue ventrale, $\mathrm{E}$, extrémité antérieure, vue ventrale. F, † immature, ovéjecteur disséqué, vue latérale droite, $\mathrm{G}, \uparrow$ mûre, vulve, ovéjecteur, début des utérus, vue ventrale, $\mathrm{H}, q$, queue, vue latérale gauche. A, B, C, éch. : $50 \mu ;$ D, F, éch. : $100 \mu$; E, G, H, éch. : $200 \mu$. 
L'un d'entre nous a récolté au Togo un certain nombre de Nématodes d'Amphibiens et parmi ceux-ci, deux paraissent particulièrement intéressants, l'un étant nouveau et l'autre n'étant connu que par la description d'un spécimen mâle.

Nous en faisons donc l'étude ci-après, les spécimens types étant déposés dans les collections du Museum National d'Histoire Naturelle de Paris.

\section{Gendria leberret Bain et Philippon, 1969}

Chez deux Dicroglossus occipitalis (Gunther) originaires de Lomé, quelques spécimens $\delta^{*}$ et $q$ que nous avons identifiés à l'espèce Gendria leberrei ont été récoltés. Cette espèce n'est connue que par un mâle, parasite de Bufo regularis Reuss en HauteVolta. Il nous a donc paru intéressant de compléter la description originale :

RÉGION CÉPHALIQUE: Bouche entourée de six lèvres portant chacune une papille labiale interne. Les labiales externes et les céphaliques ne sont pas fusionnées. Bien que sur vue apicale (fig. 1, C), les amphides simulent des labiales externes, nous pensons que ce sont bien en réalité les amphides, d'une part à cause de l'aspect en vue latérale (fig. 1, A, B), d'autre part, parce qu'elles sont en position plus dorsale. Dans notre interprétation, les 2 labiales externes seraient donc atrophiées et auraient un aspect différent des 4 labiales externes sub-médianes, fortement développées. Les 4 céphaliques sont formées de 2 parties: une partie antérieure, très fine, une partie postérieure élargie, en forme de croissant (fig. 1, A), les 3 dents pharyngiennes sont de taille inégale, la dorsale étant plus grosse que les deux latéro-ventrales (fig. 1, C).

MÂle : Corps long de $13,35 \mathrm{~mm}$, large de $250 \mu$ dans sa partie moyenne. Anneau nerveux et pore excréteur situés à $180 \mu$ et $485 \mu$ de l'apex. Deirides au même niveau que le pore excréteur, en forme de crochet. Esophage long de $485 \mu$, l'osophage musculaire étant nettement plus court que le glandulaire. Spicules : $270 \mu$; queue : $180 \mu$; Papilles caudales : 6 paires précloacales, étagées de la ventouse au cloaque, plus une grosse papille impaire en avant de l'anus ; 5 paires de post-cloacales, plus les phasmides situées en arrière de la $4^{\circ}$ paire (fig. 2).

Femelle: Corps long de $16,9 \mathrm{~mm}$ sur $350 \mu$ de large dans sa partie moyenne. Anneau nerveux, pore excréteur et deirides situés à $135 \mu, 275 \mu$ et $280 \mu$ de l'apex. Esophage long de $290 \mu$ divisé en œsophage musculaire et glandulaire (fig. 1, E).

La vulve s'ouvre à $7100 \mu$ de la pointe caudale. Ovéjecteur, dirigé vers l'avant, long de $370 \mu$ et formé de 2 parties différentes, de longueur sensiblement égale. La partie postérieure est fortement muscularisée, la partie antérieure, à parois minces, débouche sur les 2 utérus (fig. 1, F, G). Ceux-ci, opposés, renferment de très nombreux œufs dont certains sont embryonnés, hauts de $60 \mu$ sur $40 \mu$ de large. Queue longue de $240 \mu$, fortement amincie à son extrémité (fig. 1, H). 

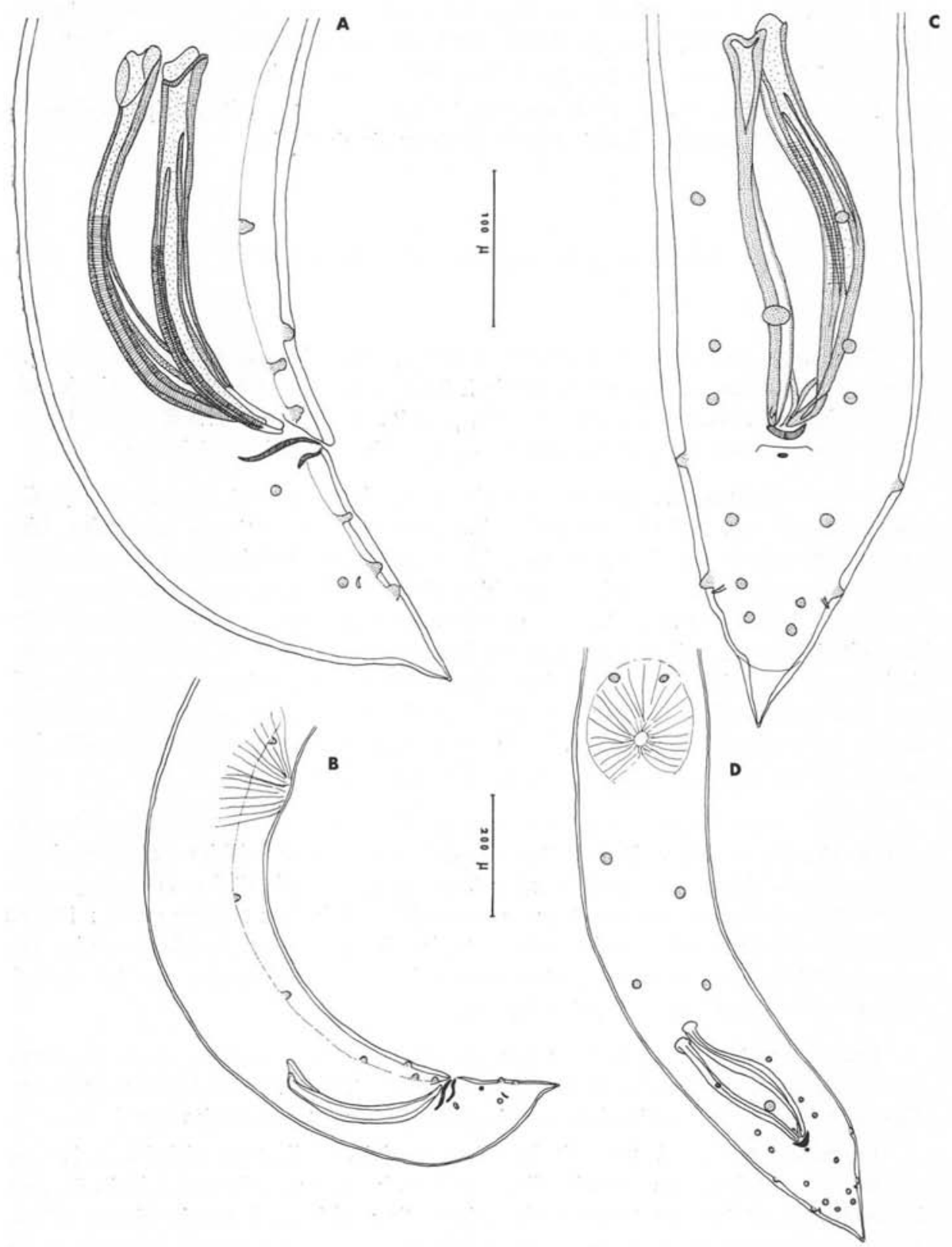

Fig. 2. - Gendria leberrei. Bain et Philippon, 1969. ơ A, B, extrémité postérieure, vue latérale droite. $\mathrm{C}, \mathrm{D}$, extrémité postérieure, vue ventrale. A, C, éch. : $100 \mu ; \mathrm{B}, \mathrm{D}$, éch. : $200 \mu$. 
Discussion : Les spécimens du Dicroglossus nous paraissent tout à fait identifiables à Gendria leberrei, malgré certaines différences (céphaliques et labiales externes fusionnées, une paire de papilles précloacales en moins) dues au fait que les auteurs n'avaient qu'un seul spécimen $\sigma^{\star}$ à leur disposition. Nous sommes en accord avec les auteurs pour ranger l'espèce dans les Quimperiidae, dont elle présente les principaux caractères.

\section{Camallanus dimitrovi r.sp.}

MATÉRIEL TYPE : $2 \delta^{\star}, 1$ ᄋ, $\mathrm{n}^{\circ} 758 \mathrm{HA}$.

HôTE : Dicroglossus occipitalis (Günther) (hôte type).

ORIGINE GÉOGRAPHIQUE : Lomé.

Localisation: Intestin grêle.

AUtre MATÉRIEl : quelques $\sigma^{*}$ et $\%$, parasites de 5 autres Dicroglossus occipitalis originaires de la même région $\left(\mathrm{n}^{\circ} 23-26-36-37\right) ; 1 \delta$, 1 , parasites de l'intestin grêle d'une Rana galamensis Dumeril et Bibron originaire de Klouto ; 1 q parasite de l'ampoule rectale d'un Dicroglossus occipitalis de Lomé.

Description : Nématodes de taille moyenne, la femelle étant presque deux fois plus grande que le mâle. Corps aminci aux deux extrémités, sur une longueur d'environ le sixième du corps à l'extrémité postérieure.

RÉGION CÉPHALIQUE: Capsule buccale presqu'aussi haute que large, limitée par deux valves latérales articulées, à parois très épaisses, 19 à 21 baguettes chitinoïdes longitudinales présentes sur chaque valve. Aucune n'atteint la partie distale de la valve. Chaque valve porte à son extrémité antérieure, de chaque côté du plan frontal, deux formations chitinoïdes. A la jonction des valves, dans le plan médian, se trouvent 2 grosses pièces chitinoïdes en forme de trident (fig. 3, B, C). Le fond de la cavité buccale est limité par un épais collier chitinoïde. En vue apicale, on observe 6 papilles labiales internes situées de part et d'autre de l'ouverture buccale, puis plus extérieurement 4 papilles labiales externes en forme de languettes, enfin les 4 céphaliques en forme de coupole. Les amphides sont très petites (fig. 3, D).

MÂle : Corps long de $13,7 \mathrm{~mm}$, large de $380 \mu$ dans sa partie moyenne, de $300 \mu$ dans sa partie postérieure. Capsule buccale ; $145 \mu$ de haut sur $155 \mu$ de large au sommet, $105 \mu$ à sa base. Branches du trident : $95 \mu$ de long. Anneau nerveux, pore excréteur, deirides situées respectivement à $220 \mu, 425 \mu$ et $435 \mu$ de l'apex. Esophages musculaire et glandulaire longs respectivement de $400 \mu$ et $475 \mu$.

Queue, longue de $200 \mu$, incurvée ventralement, à terminaison arrondie. Ailes caudales hautes de $800 \mu$, larges au maximum de $150 \mu$. Présence de 14 paires de papilles: 7 précloacales, portant un long pédoncule, 2 entourant le cloaque, 5 postcloa- 
cales, les 3 premières étant groupées. Les phasmides sont situées juste en avant de la $4^{\circ}$ paire. Spicule droit, long de $500 \mu$ sur $9 \mu$ de large dans sa partie moyenne. Sa partie postérieure, longue de $65 \mu$, prend la forme d'un pied et se termine par 2 bran-

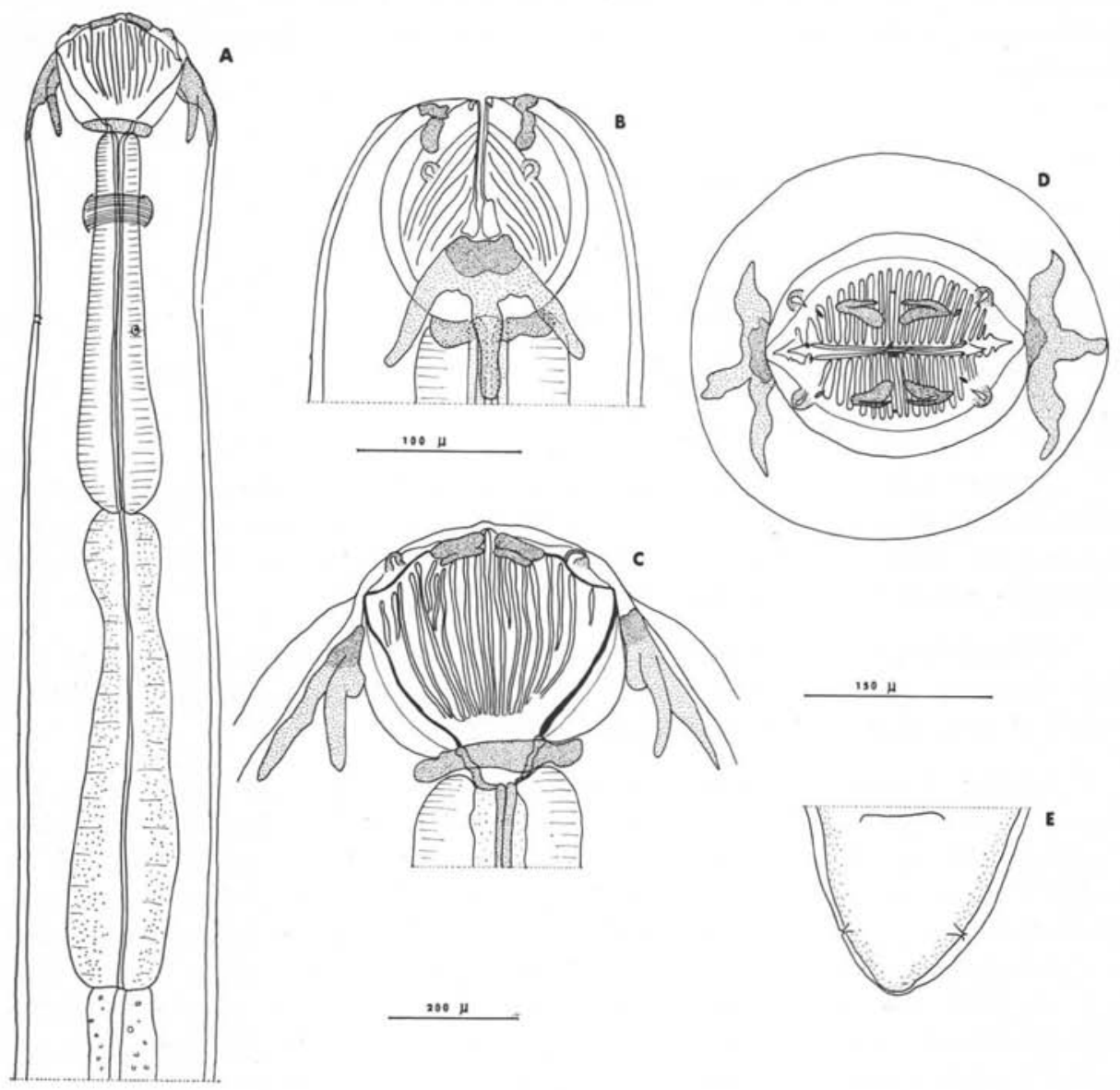

FIG. 3. - Camallanus dimitrovi n.sp., A, ㅇ, extrémité antérieure, vue latérale gauche. $\mathrm{B}, \mathrm{C}, \mathrm{D}, \delta^{\hat{\sigma}}$, tête successivement en vues médiane, latérale et apicale. $\mathrm{E}, \stackrel{+}{+}$ queue, vue ventrale. A, éch. : $200 \mu$; B, C, D, éch. : $100 \mu$; E, éch. : $150 \mu$.

ches enfermées dans une gaine (fig. 4, E). Spicule gauche long de $350 \mu$ et large de $7 \mu$ dans sa partie moyenne. Il n'est possible de l'observer qu'après dissection car, en place, il reste collé sur le spicule droit et est invisible, étant moins large et moins chitinisé que ce dernier. Sa partie postérieure bifide est légèrement coudée ventralement et les pointes sont enfermées dans une gaine (fig. 4, D). 


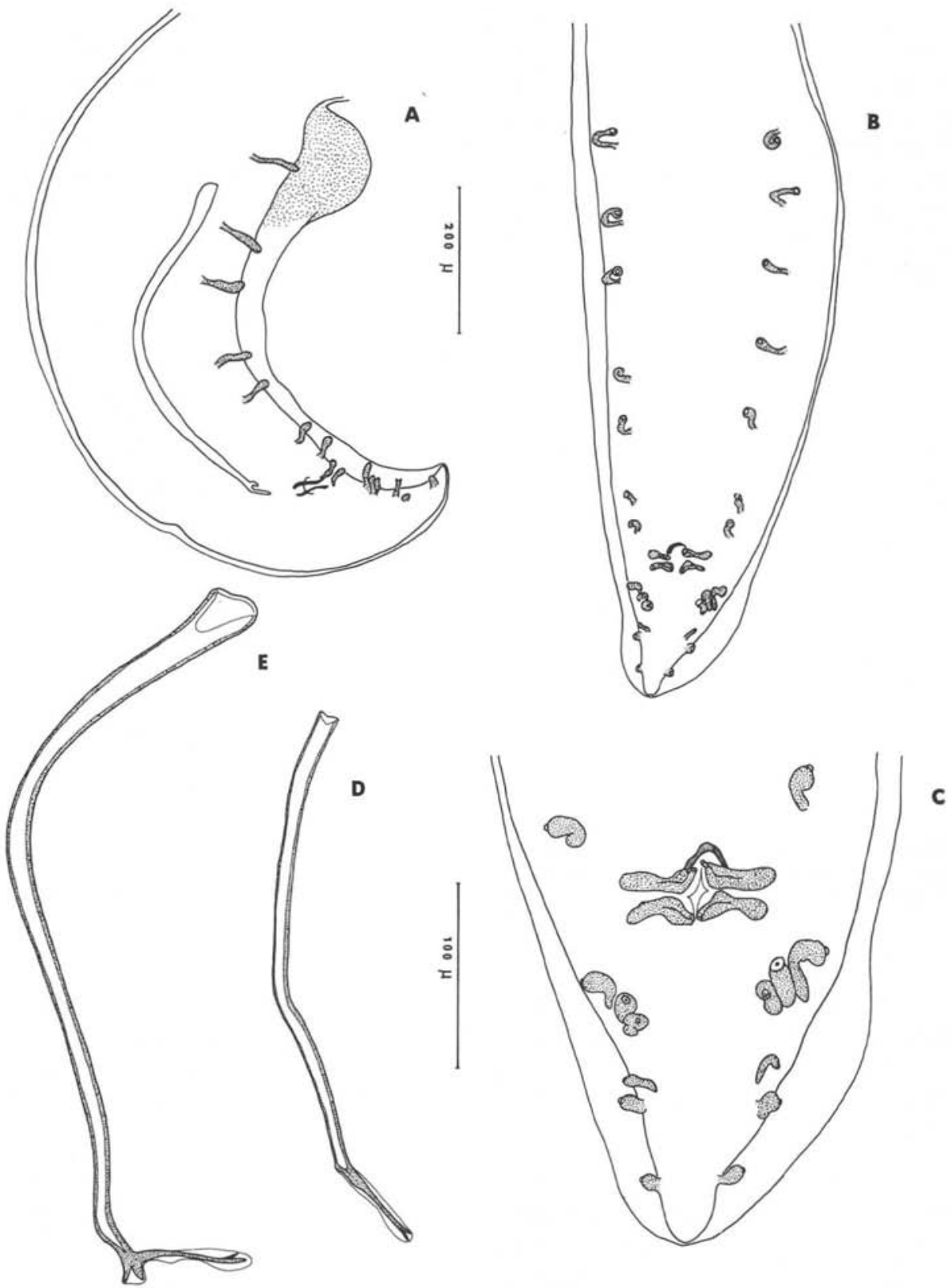

Fig. 4. - Camallanus dimitrovi n.sp., ơ, A, extrémité postérieure, vue latérale droite. $\mathrm{B}$, extrémité postérieure, vue ventrale. $\mathrm{C}$, queue, vue ventrale. $\mathrm{D}$, spicule gauche disséqué. $\mathrm{E}$, spicule droit disséqué. A, B, éch. : $200 \mu ; \mathrm{C}, \mathrm{D}, \mathrm{E}$, éch. : $100 \mu$. 
Femelle : Corps long de 21,6 mm, large de $450 \mu$ dans sa partie moyenne et de $350 \mu$ dans sa partie postérieure. Capsule buccale haute de $180 \mu$ sur $200 \mu$ de large au sommet et $110 \mu$ à sa base. Branches du trident : $150 \mu$ de long. Anneau nerveux, pore excréteur et deirides situés respectivement à $280 \mu, 470 \mu$ et $480 \mu$ de l'apex. Esophages musculaire et glandulaire longs respectivement de $590 \mu$ et $740 \mu$ (fig. 3, A). Vulve située à $8,7 \mathrm{~mm}$ de l'apex. Ovéjecteur musculaire dirigé vers l'arrière ; les deux branches utérines, à parois minces, contiennent un très grand nombre d'œufs. Queue à extrémité arrondie, longue de $140 \mu$. Phasmides situées à $50 \mu$ de l'extrémité caudale (fig. 3, E).
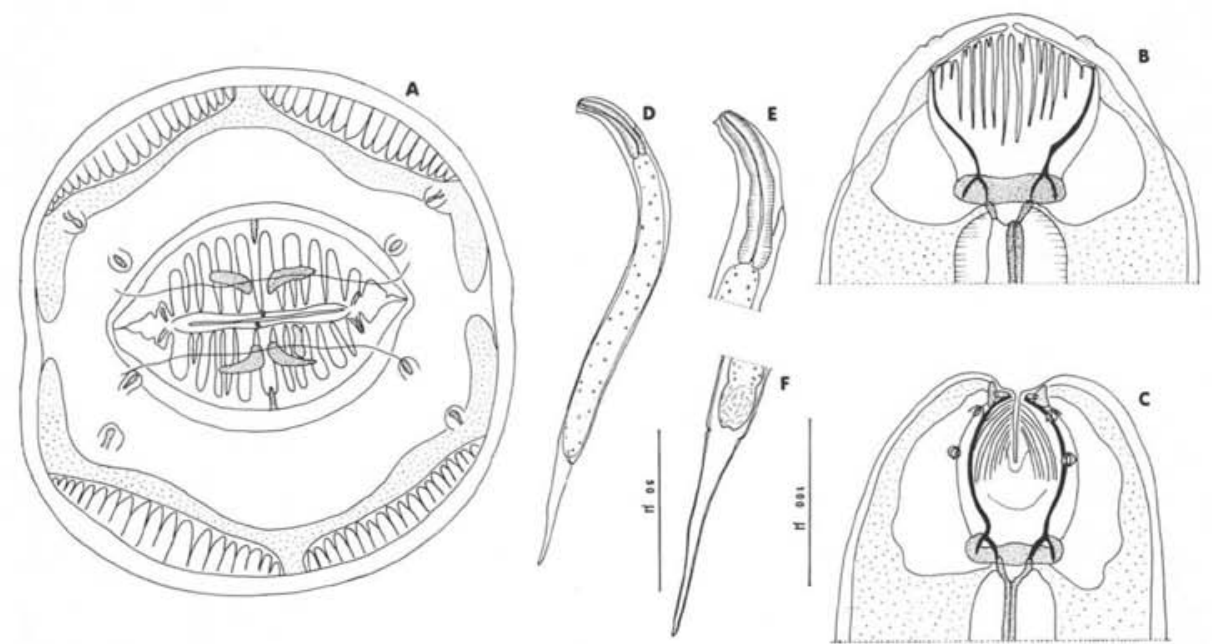

FIG. 5. - Camallanus dimitrovi, n.sp. Quatrième stade larvaire. 우 A, B, C, tête successivement en vues apicale, latérale et médiane. Premier stade larvaire. D, E, F, successivement animal entier, vue latérale droite; extrémité antérieure, vue latérale droite ; extrémité postérieure, vue ventrale. A, B, C, éch. : $100 \mu$; D, E, F, éch. : $50 \mu$.

Les spécimens femelles d'un autre Dicroglossus $\left(\mathrm{n}^{\circ} 36\right)$ étaient vivipares. Tous les autres caractères concordant avec la description des types, nous pensons qu'il s'agit de la même espèce. La segmentation des œufs n'était probablement pas achevée chez la femelle type, ceci étant lié au fait qu'elle est nettement plus petite que les autres femelles qui mesurent plus de $30 \mathrm{~mm}$.

LARVES DU PREMIER STADE : Elles mesurent $310 \mu$ de long sur $15 \mu$ de large dans leur partie moyenne (fig. 5, D). Queue longue de $67 \mu$, très effilée. Phasmides bien visibles (fig. 5, F). Tête portant une petite dent dorsale. Esophage : $50 \mu$ de long. Pore excréteur situé à $32 \mu$ de l'apex (fig. 5, E).

QUATRIÈme STADE LARVAIRE FEMElle: Dans le tube $\mathrm{n}^{\circ} 36$, nous avons trouvé un quatrième stade larvaire femelle que nous décrivons ci-après. 
Principales mensurations: Corps long de $6,75 \mathrm{~mm}$, large de $180 \mu$ dans sa partie moyenne. Anneau nerveux, pore excréteur et deirides situés respectivement à $155 \mu, 320 \mu$ et $320 \mu$ de l'apex. Esophage long de $830 \mu$; œsophage musculaire, $380 \mu$; glandulaire $450 \mu$. Queue longue de $105 \mu$. Phasmides bien visibles. Trois pointes caudales.

RÉGION CÉPHAliQue: Elle est proche de celle de l'adulte mais dépourvue de tridents médians. La surface des 2 valves porte une quinzaine de baguettes chitinoïdes, dont certaines sont très courtes et dont aucune n'atteint la partie distale de la valve (fig. 5, B, C). Les 4 formations antérieures situées de chaque côté du plan frontal sont présentes.

En vue apicale, la tête porte 6 papilles labiales internes situées de part et d'autre de l'ouverture buccale, 4 papilles labiales externes aussi développées que les 4 papilles céphaliques. Les amphides sont en forme de languettes (fig. 5, A).

Discussion : D’après la classification de Chabaud (in Grassé, 1965), nos spécimens présentent les principaux caractères du genre Camallanus, Railliet et Henry, 1915 et, en particulier, la présence d'une capsule buccale, pourvue de deux tridents médians et formée par 2 valves, sans cavité buccale en arrière de celles-ci.

A notre connaissance, treize espèces sont actuellement décrites chez les Amphibiens. Ces espèces sont caractérisées par quatre éléments que l'on rencontre avec une fréquence plus élevée que chez les Camallanus de Poissons. Ces 4 éléments sont les suivants :

(1) Les trois premières paires de papilles postcloacales sont groupées et nettement séparées de la ou des paires suivantes. Onze espèces possèdent ce caractère. Il est absent chez $C$. cynophylectis Sahay, 1966. Chez C. multiruga Walton, 1932, la description ne permet pas de préciser la disposition des papilles caudales.

(2) L'extrémité postérieure du spicule droit est fortement coudée et prend la forme d'un pied. Sept espèces possèdent ce caractère, absent chez cinq espèces et non visible sur la description de $C$. multiruga.

(3) La position relativement antérieure de la vulve. Elle est prééquatoriale chez 8 espèces, sub-équatoriale chez 3 et post-équatoriale chez 2 .

(4) La viviparité. Toutes les espèces chez lesquelles ce caractère a été indiqué (soit 8) sont vivipares.

- 5 espèces présentent au moins 1 de ces caractères, 3 en présentent 2 et 5 en possèdent 4.

Chez les 32 Camallanus de Poissons décrits, nous avons trouvé 3 fois le premier caractère, jamais le deuxième, et 6 fois le troisième.

Nous ne pouvons donner le chiffre exact pour le dernier caractère qui n'a pas toujours été précisé par les auteurs.

Chez les Camallanus de Poissons, qui possèdent les caractères précités, ceux-ci ne sont jamais liés, chaque espèce n'en présentant qu'un seul à la fois. 
Il apparaît donc que les Camallanus d'Amphibiens forment un groupe à part, individualisé dans une grande mesure de celui des Camallanus de Poissons et paraissent plus évolués (papilles groupées, forme du spicule, viviparité) que ce dernier.

Il nous paraît cependant prématuré de créer un nouveau sous-genre pour ce groupe.

Les spécimens de Dicroglossus se rangent aisément dans le groupe des Camallanus d'Amphibiens, puisqu'ils possèdent les 4 caractères précités. Les espèces les plus proches sont $C$. baylisi, Karve, 1930, parasite de Rana tigrina en Asie, C. inglisi Agrawal, 1967, parasite de $R$. tigrina en Inde et $C$. thapari Gupta, 1959, parasite de $R$. tigrina au Pakistan. Nos spécimens se différencient aisément de toutes ces espèces par le nombre nettement plus élevé de baguettes céphaliques. De plus, chez $C$. inglisi, la disposition des papilles précloacales est différente; chez $C$. thapari, le trident est moins développé et le $\delta^{t}$ et la ㅇ possèdent 2 pointes caudales, enfin chez $C$. baylisi, le plus proche de nos spécimens pour la disposition des papilles caudales, la queue du $\sigma$ est bifide.

Nous pensons donc que nos spécimens sont nouveaux et nous les nommons Camallanus dimitrovi n. sp. en hommage au Professeur Dimitrov.

\section{Bibliographie}

Agrawal (V.), 1967. - Some new Camallanoidea (Spirurida) Nematodes from fishes, Amphibians and Reptiles. Ann. Parasit. hum. comp., 42 (3), 327-342.

Bain (O.) et Philippon (B.), 1969. - Recherche sur des larves de Nématodes Ascaridida trouvées chez Simulium damnosum. Ann. Parasit. hum. comp., 44 (2), 147-156.

Chabaud (A.-G.), 1965. - Sous-ordre des Camallanina: 1030-1043, in P.-P. Grasse, Traité de Zoologie, 4 (2-3), Masson et $C^{1 e}$, édit., Paris, $1497 \mathrm{p}$.

GuPTA (S. P.), 1959. - Nematode parasites of vertebrates of East Pakistan. Three Camallanides from fishes, amphibia and reptiles. Canad. J. Zool., 37, 771-779.

KarVe (J. N.), 1930. - Some parasitic nematodes of frogs and toads. Ann. Trop. Med. and Parasit., 24 (4), 481-491.

RaIlliet (A.) et Henry (H.), 1915. - Sur les Nématodes du genre Camallanus Railliet et Henry, 1915 (Cucullanus auct., non Mueller, 1777). Bull. Soc. Path. Exot., 8, 446-452.

SAHAY (U.), 1966. - On a new species of Camallanus Railliet and Henry, 1915, from Rana cynophylectis. Ind. J. Helm., 18 (1), 53-56.

Walton (A. C.), 1932. - A new Nematode (Camallanus multiruga sp.n.) parasitic in a West african frog. Ann. Mag. Natur. Hist., 10 (9), 151-154. 